\title{
High Frequency of $45, X / 46, X Y$ mosaicism carring a structurally abnormal $Y$ chromosome in patients with Y chromosome microdeletions: a 8-year period retrospective study
}

Hong-Ge Li ( $\square$ mylove6000@163.com )

Zhejiang University

\section{Research}

Keywords: 45,X/46,XY mosaicism, Y chromosome microdeletions, Azoospermia Factor (AZF), Structural abnormalities of $Y$ chromosome, spermatogenic failure

Posted Date: July 22nd, 2020

DOI: https://doi.org/10.21203/rs.3.rs-44357/v1

License: @ (i) This work is licensed under a Creative Commons Attribution 4.0 International License. Read Full License 


\section{Abstract}

Background: Structural abnormalities of $Y$ chromosome are commonly described in associated with the occurrence of a 45,X/46,XY chromosomal mosaicism. In recent years, evidences of an association between $Y$ chromosome microdeletions and 45,X/46,XY mosaicism had been investigated, and 45,X/46,XY mosaicism was found to be particularly common in patients with $Y$ chromosome microdeletions. The aim of our study was to investigate the presence of $45, X / 46, X Y$ mosaicism carrying a structurally abnormal $Y$ chromosome in patients with $\mathrm{Y}$ chromosome microdeletions.

Results: A 8-year retrospective study was conducted on 6545 infertile men with nonobstructive azoospermia or oligozoospermia. A total of 19 patients with $45, \mathrm{X} / 46, \mathrm{XY}$ mosaicism or its variants were found, of which $78.95 \%(15 / 19)$ had a structural $Y$ chromosome abnormalities in $46, X Y$ cell line. Thirteen of $19(68.42 \%$, 13/19) patients with $45, X / 46, X Y$ mosaicism had $Y$ chromosome microdeletions, and $12 / 13(92.31 \%)$ of them exhibited a structurally abnormal $Y$ chromosome.

Conclusions: Our results were consistent with previous studies that a high frequency of $45, X / 46, X Y$ mosaicism or its variants were detected in patients with $Y$ chromosome microdeletions. Different from previous studies, we found that $45, \mathrm{X} / 46, \mathrm{XY}$ mosaicism in patients with $\mathrm{Y}$ chromosome microdeletions almost all exhibited a structurally abnormal $\mathrm{Y}$ chromosome.

\section{Background}

Chromosomal abnormalities and $Y$ chromosome microdeletions are considered to be the two more common genetic causes of spermatogenic failure. The mosaic $45, \mathrm{X} / 46, \mathrm{XY}$ karyotype is a common sex chromosomal abnormality associated with male infertility. Structural abnormalities of $Y$ chromosome are estimated to affect $10-20 \%$ of men with non-obstructive azoospermic infertility[1], which are commonly described in associated with the occurrence of a $45, X / 46, X Y$ chromosomal mosaicism due to a probable mitotic instability of the abnormal chromosome[2]. A previous report showed that more than half of the patients with $Y$ chromosome structural abnormalities might present with $45, \mathrm{X}$ mosaic cell lines [3]. $Y$ chromosome microdeletions are associated with severe spermatogenic failure and represent the most frequent molecular genetic cause of azoospermia and severe oligozoospermia [4]. Azoospermia Factor (AZF) region is responsible for the $Y$ chromosome microdeletions. This region located on the long arm of $Y$ (Yq11.23) and was mapped to three non-overlapping regions described as AZFa, AZFb, and AZFc[5]. The frequency of $\mathrm{Y}$ chromosome microdeletions was reported to be in the range of $3-55 \%$ according to previous studies with an average frequency of $7 \%[6]$.

In recent years, evidences of an association between $\mathrm{Y}$ chromosome microdeletions and the formation of $45, X / 46, X Y$ mosaicism had been investigated, and found that $45, X / 46, X Y$ mosaicism to be particularly common in patients with $Y$ chromosome microdeletions. The aim of our study was to investigate the presence of $45, X / 46, X Y$ mosaicism carryiny a structurally abnormal $Y$ chromosome in patients with $Y$ chromosome microdeletions. Here, we retrospectively reviewed a single center experience over a 8-year period (2012-2019) at a university teaching hospital in southern China. Our study included 19 clinically well-characterized mosaic 
patients whose karyotype consisted of a 45,X cell line and a second cell line containing a normal or an abnormal Y chromosome.

\section{Material And Methods}

\section{Subjects}

We conducted a retrospective study of infertile men with nonobstructive azoospermia or oligozoospermia from male outpatients of the Women's Hospital of Zhejiang University between January 1 st 2011 and December 31st 2019. A total of 6,545 cases of infertility men were recruited in the study. The patients' ages ranged from 17 to 63 (mean $\pm S D, 31.45 \pm 5.29$ ) years, and infertility lasted from 2 to 21 years old. Karyotype analysis and $Y$ chromosome microdeletions were performed in all patients. This study was approved by the Scientific Research Ethics Committee of the Women's Hospital of Zhejiang University. This was a retrospective study of the clinical database with no intervention and no informed consent was required. All the methods used in the study followed the current approved guidelines.

\section{Karyotype Analysis}

Metaphase chromosomes with targeted 400-band level were performed as previously described [7]. Briefly, about $1 \mathrm{~mL}$ peripheral blood was inocubated aseptically into culture bottle containing of $5 \mathrm{~mL}$ lymphocyte culture solution in $5 \% \mathrm{CO}_{2}$ incubator at $37^{\circ} \mathrm{C}$ for $72 \mathrm{~h}$. Then $20 \mu \mathrm{g} \mathrm{ml} l^{-1}$ colchicine was added to the culture system half an hour before the termination of cell culture to arrest the chromosomes at metaphase. G-banding of metaphase chromosomes were obtained by hypotension, fixation, trypsinization, Giemsa staining, and so forth. At least 30 metaphases were counted and 5 metaphases were analyzed for each sample by two certified physicians according to the International System for Human Cytogenetic Nomenclature guidelines (ISCN, 2016, 5th edition) [8].

\section{Y Chromosome Microdeletions}

Y chromosome microdeletions were performed by multiplex PCR amplification as previously described [7]. Six specific sequence-tagged sites (STS) that recommended by the European Academy of Andrology (EAA) and the European Molecular Genetics Quality Network (EMGQ) were used for the detection of $Y$ chromosome microdeletions, including sY84 and sY86 for AZFa, sY127 and sY134 for AZFb, sY254 and sY255 for AZFc. The Human $Y$ Chromosome Microdeletion Gene Detection Kit was used for the detection of $Y$ chromosome microdeletion. Multiplex PCR amplification was performed on the LightCycler 480 II thermocycler. PCR was performed with the following thermal cycling conditions: $95^{\circ} \mathrm{C}$ for $3 \mathrm{~min}$, then 10 cycles at $95^{\circ} \mathrm{C}$ for $15 \mathrm{~s}, 63^{\circ} \mathrm{C}$ for $20 \mathrm{~s}, 72^{\circ} \mathrm{C}$ for $20 \mathrm{~s}$, followed by 30 cycles at $95^{\circ} \mathrm{C}$ for $15 \mathrm{~s}, 63^{\circ} \mathrm{C}$ for $32 \mathrm{~s}, 72^{\circ} \mathrm{C}$ for $20 \mathrm{~s}$, and a final extension at $72^{\circ} \mathrm{C}$ for $10 \mathrm{~min}$. An STS marker was considered to be deleted only after at least two failed PCR amplification attempts with single primer pairs.

\section{Results}

A total of 19 patients with $45, \mathrm{X} / 46, \mathrm{XY}$ mosaicism or its variants were detected in 6545 male patients, with an incidence rate of $0.29 \%$ (19/6545). The results of karyotype analysis and AZF microdeletions for the 19 
patients are summarized in Table 1. Of these 19 patients with 45,X/46,XY mosaicism, 78.95\% (15/19) had a structurally abnormal $Y$ chromosome in the $46, X Y$ cell line and $21.05 \%(4 / 19)$ had pure $45, X / 46, X Y$ mosaicism. All of these patients $(100.00 \%, 15 / 15)$ with a structurally abnormal $Y$ chromosome and three patients $(75.00 \%, 3 / 4)$ with pure $45, X / 46, X Y$ mosaicism exhibited azoospermia, while the remaining one patients with pure $45, X / 46, X Y$ mosaicism exhibited oligozoospermia. Thirteen of 19 patients $(68.42 \%, 13 / 19)$ with 45,X/46,XY mosaicism had AZF microdeletions, including 11 patients $(84.62 \%, 11 / 13)$ with AZFb + C microdeletions and 2 patients $(15.38 \%, 2 / 13)$ with AZFc microdeletions. In the present study, $92.31 \%(12 / 13)$ of $45, X / 46, X Y$ mosaicism in patients with $Y$ chromosome microdeletions exhibited a structural $Y$ chromosome abnormalities in 46,XY cell line; six with Yqh-, five with del(Y) (q12), and one with $Y \leq 21$. All patients $(100.00 \%$, $11 / 11)$ with AZFb $+c$ microdeletions and one of two patients $(50 \%, 1 / 2)$ with AZFc microdeletions exhibited a structurally abnormal Y chromosome. Moreover, a totla of 315 patients with AZF microdeletion were identified in this study. The pattern/number and characteristic of the patients showing these deletions were shown in Table 2. Only the deletions of AZFc region and $\mathrm{AZFb}+\mathrm{c}$ region invovled $45, \mathrm{X} / 46, \mathrm{XY}$ mosaicism, including eleven of $60(18.33 \%, 11 / 60)$ men with the AZFb $+c$ deletion and two of $208(0.96 \%, 2 / 208)$ men with the AZFc deletion. 
Table 1

The results of AZF microdeletions in men with 45, $\mathrm{X} / 46, \mathrm{XY}$ mosaicism or its variants reported in the literature.

\begin{tabular}{|c|c|c|c|c|}
\hline $\begin{array}{l}\text { No. of } \\
\text { Patients }\end{array}$ & Age & Karyotype Results & $\begin{array}{l}\text { Microdeletions } \\
\text { Results }\end{array}$ & $\begin{array}{l}\text { Clinical } \\
\text { diagnosis }\end{array}$ \\
\hline Case 1 & 24 & $45, X[12] / 46, X, \operatorname{del}(Y)(q 12)[8]$ & $A Z F b+c$ & azoospermia \\
\hline Case 2 & 36 & $45, X[25] / 46, X, \operatorname{del}(Y)(q 12)[5]$ & $\mathrm{AZFb}+\mathrm{C}$ & azoospermia \\
\hline Case 3 & 31 & $45, X[26] / 46, X Y, Y \leq 21[14]$ & $\mathrm{AZFb}+\mathrm{C}$ & azoospermia \\
\hline Case 4 & 30 & $45, X[34] / 46, X, Y q h-[16]$ & $\mathrm{AZFb}+\mathrm{c}$ & azoospermia \\
\hline Case 5 & 27 & $46, X, \operatorname{del}(Y)(q 12)[41] / 45, X[10]$ & $\mathrm{AZFb}+\mathrm{C}$ & azoospermia \\
\hline Case 6 & 36 & $46, X, \operatorname{del}(Y)(q 12)[17] / 45, X[13]$ & $A Z F b+c$ & azoospermia \\
\hline Case 7 & 27 & $46, X, \operatorname{del}(Y)(q 12)[8] / 45, X[7]$ & $A Z F b+c$ & azoospermia \\
\hline Case 8 & 33 & $46, X, Y q h-[21] / 45, X[9]$ & $\mathrm{AZFb}+\mathrm{C}$ & azoospermia \\
\hline Case 9 & 30 & $46, X, Y q h-[23] / 45, X[7]$ & $\mathrm{AZFb}+\mathrm{C}$ & azoospermia \\
\hline Case 10 & 34 & $46, X, Y q h-[44] / 45, X[6]$ & $A Z F b+c$ & azoospermia \\
\hline Case 11 & 27 & $46, X, Y q h-[45] / 45, X[5]$ & $A Z F b+c$ & azoospermia \\
\hline Case 12 & 29 & $45, X[6] / 46, X Y[16]$ & AZFc & azoospermia \\
\hline Case 13 & 28 & $46, X, Y q h-[14] / 45, X[6]$ & AZFc & azoospermia \\
\hline Case 14 & 29 & $45, X[6] / 46, X Y[44]$ & Normal & oligozoospermia \\
\hline Case 15 & 28 & $46, X,+\operatorname{mar}[22] / 46, X, \operatorname{del}(Y)(q 12)[8] / 45, X[2]$ & Normal & azoospermia \\
\hline Case 16 & 31 & $46, X,+\operatorname{mar}[36] / 45, X[11] / 46, X Y[3]$ & Normal & azoospermia \\
\hline Case 17 & 28 & $46, X, \operatorname{idic}(Y)(p 11.3)[45] / 45, X[4]$ & Normal & azoospermia \\
\hline Case 18 & 40 & $46, X X[16] / 47, X X Y[8] / 45, X[3] / 46, X Y[2] / 48, X X X Y[1]$ & Normal & azoospermia \\
\hline Case 19 & 24 & $46, \mathrm{XY}, 1 \mathrm{qh}+[28] / 45, \mathrm{X}, 1 \mathrm{qh}+[22]$ & Normal & azoospermia \\
\hline
\end{tabular}


Table 2

The pattern/number and characteristic of patients with AZF microdeletions in the study.

\begin{tabular}{|lllll|}
\hline \multicolumn{2}{|c}{ AZF Microdeletions } & \multicolumn{3}{c|}{ Karyotype Results } \\
\hline Deletion Patterns & No. of Patients & Percentage & Mosaic 45,X/46,XY & Other Karyotype \\
\hline sY127 & 1 & $0.32 \%$ & $0(0.00 \%)$ & $1(100.00 \%)$ \\
\hline sY84 & 1 & $0.32 \%$ & $0(0.00 \%)$ & $1(100.00 \%)$ \\
\hline sY134 & 2 & $0.63 \%$ & $0(0.00 \%)$ & $2(100.00 \%)$ \\
\hline AZFc + sY134 & 3 & $0.95 \%$ & $0(0.00 \%)$ & $3(100.00 \%)$ \\
\hline AZFa & 11 & $3.49 \%$ & $0(0.00 \%)$ & $11(100.00 \%)$ \\
\hline AZFa +b+c & 12 & $3.81 \%$ & $0(0.00 \%)$ & $12(100.00 \%)$ \\
\hline AZFb & 17 & $5.40 \%$ & $0(0.00 \%)$ & $17(100.00 \%)$ \\
\hline AZFb +c & 60 & $19.05 \%$ & $11(18.03 \%)$ & $49(81.67 \%)$ \\
\hline AZFc & 208 & $66.03 \%$ & $2(0.96 \%)$ & $206(99.04 \%)$ \\
\hline Total & 315 & $100.00 \%$ & $13(4.13 \%)$ & $302(95.87 \%)$ \\
\hline
\end{tabular}

\section{Discussion}

The prevalence of $45, X / 46, X Y$ mosaicisms or its variants in this male infertility study was $0.29 \%(19 / 6545)$ (approximately 29/10,000), which was consistent with previous studies $(0.27 \%)$ [9]. However, this is much higher than its incidence $(1.5 / 10,000)$ in newborns [10], indicating that $45, X / 46, X Y$ mosaicisms are common chromosomal aberrations associated with male infertility. In this study, we analysed 6545 infertile men and found that $68.42 \%(13 / 19)$ of patients with $45, X / 46, X Y$ mosaicism had AZF microdeletions. In previous studies, Li et al investigated 5269 cases of infertility men and found that $71.43 \%(10 / 14)$ of patients with 45,X/46,XY mosaicism exhibited AZF microdeletions [9]. Pan et al detected 5235 male patients with primary infertility and reported that $83.3 \%$ (5/6) patients with mosaic karyotype $45, X / 46, X Y$ had AZF deletions [11]. dos Santos AP et al studied 15 patients with mosaicism and found that approximately 40\% (6/15) patients with AZF deletions had mosaic karyotype 45,X/46,XY [12]. The prevalence of $Y$ chromosome microdeletions in patients with $45, X / 46, X Y$ mosaicism or its variants was different from other reports, main cause of which might was the sample size. However, our results were consistent with previous studies that a high frequency of $\mathrm{Y}$ chromosome microdeletions was detected in patients with $45, \mathrm{X} / 46, \mathrm{XY}$ mosaicism or its variants.

In the study, $78.95 \%(15 / 19)$ patients with $45, X / 46, X Y$ mosaicism had a structurally abnormal $Y$ chromosome in the $46, X Y$ cell line, while $21.05 \%$ (4/19) had pure 45,X/46,XY mosaicism. Different from previous studies, we found that $45, X / 46, X Y$ mosaicism in patients with $Y$ chromosome microdeletions almost all exhibited a structurally abnormal $Y$ chromosome. In this study, $92.31 \%$ (12/13) of $45, X / 46, X Y$ mosaicism in patients with $Y$ chromosome microdeletions exhibited a structural $Y$ chromosome abnormalities in $46, X Y$ cell line. Li et al found 10 patients with 45,X/46,XY mosaicism had AZF microdeletions, and $40.00 \%(4 / 10)$ of them exhibited a structurally abnormal $\mathrm{Y}$ chromosome[9]. dos Santos AP et al reported a group of six patients with $45, \mathrm{X} / 46, \mathrm{XY}$ 
mosaicism had AZF microdeletions, and $66.67 \%(4 / 6)$ of them an abnormal $Y$ chromosome had been detected in cytogenetic analysis [12]. Pan et al reported 8 patients with mosaic karyotype $45, X / 46, X Y$ had AZF deletions, and $37.5 \%$ (3/8) of them had an structurally abnormal $Y$ chromosome [11]. The prevalence of $45, X / 46, X Y$ mosaicism carrying a structurally abnormal $Y$ chromosome in patients with $Y$ chromosome microdeletions was much higher than other reports. Karyotyping is a reliable technique for the identification of most chromosomal abnormalities, but it cannot detect subtle variations in chromosomal structure, only detecting unbalanced anomalies of at least 5-20 Mb[13]. Patients with the del(Y)(q12) karyotype had a structural deletion on the $Y$ chromosome, which is close to the AZF region. In addition, our previous study confirmed that $Y$ chromosome microdeletions only involved $Y$ chromosome polymorphic variants (especially Yqh- and $Y \leq 21$ variants) and had no relationship with other chromosome polymorphisms[8]. There were six patients with Yqh-, five with del(Y) (q12), and one with $Y \leq 21$ in the study, which might be the cause of the high frequency of structural $Y$ chromosome abnormalities in patients with $Y$ chromosome microdeletions. In addition, only one of 13 individuals with an apparently normal $Y$ chromosome had AZF microdeletions in this study. This frequency (1/13) was lower than that found by Alvarez-Nava et al (3/11) [14] and lower than that found by Patsalis et al (4/7) [15]. Taken together these findings suggest that $8 / 31(25.81 \%)$ individuals with $45, X / 46, X Y$ and apparently normal $Y$ chromosome may have $Y$ microdeletions.

In the present study, the most frequent microdeletions were detected in the AZFc region, followed by the deletion of the $A Z F b+c$ region. Consistent with previous researches, we found only the deletions of AZFc region and $\mathrm{AZFb}+\mathrm{c}$ region invovled the $45, \mathrm{X} / 46, \mathrm{XY}$ mosaicism, and deletion of $\mathrm{AZFb}+\mathrm{c}$ region might also make men more likely to lose their $Y$ chromosomes. Several studies have found AZFc deletion to be a premutation for $45, X$ and for the mosaic phenotype $45, X / 46, X Y[16,17]$. In this study, only $0.96 \%$ of men with the AZFc deletion and $18.33 \%$ of men with the AZFb + c deletion involved $45, \mathrm{X} / 46, \mathrm{XY}$ mosaicism, of which AZFb $+c$ microdeletions and AZFc microdeletions accounted for $84.62 \%(11 / 13)$ and $15.38 \%(2 / 13)$, respectively. Hopps et al[18] reported that one out of $25(1 / 25,4.0 \%)$ men with AZFc deletion and three out of $12(3 / 12,25.0 \%)$ men with AZFb $+c$ deletions had 45,X/46,XY mosaicism. Li et al reported that ten cases $(71.43 \%, 10 / 14)$ of $45, X$ mosaicism exhibiting AZF microdeletions, including two of ten $(20.0 \%, 2 / 10)$ with AZFc deletions and the other eight $(80.0 \%, 8 / 10)$ with $A Z F b+c$ deletions. Aydemir et al[19] documented a rare case of $45, X / 46, X Y$ mosaicism with deletion of the AZFb $+c$ region. Pan et al [11] reported all of five male patients $(100.0 \%, 5 / 5)$ with a mosaic karyotype $45, X / 46, X Y$ had AZFb + c deletions. However, Kleiman SE et al [20] reported that $10.29 \%(7 / 68)$ men with the AZFb + c deletion and $4.76 \%(1 / 21)$ men with AZFb deletion was found to have the $45, \mathrm{X} / 46, \mathrm{XY}$ karyotype, which was the only report about the deletions of AZFb region invovled the $45, X / 46, X Y$ mosaicism. Larger sample size and more data are needed to further prove whether AZFb microdeletion will lead to the ocure of $45, X / 46, X Y$ mosaicism.

\section{Conclusions}

Our results were consistent with previous studies that high frequency of $\mathrm{Y}$ chromosome microdeletions were detected in patients with $45, X / 46, X Y$ mosaicism or its variants, supporting the conclusions that microdeletions in the long arm of $Y$ chromosome might be associated with $Y$ chromosomal instability leading to the formation of $45, X$ cell lines. Different from previous studies, we found that $45, X / 46, X Y$ mosaicism in patients with $\mathrm{Y}$ chromosome microdeletions almost all exhibited a structurally abnormal $\mathrm{Y}$ chromosome. 


\section{Declarations}

\section{Ethics approval and consent to participate}

This study was approved by the Scientific Research Ethics Committee of the Women's Hospital of Zhejiang University. This was a retrospective study of the clinical database with no intervention and no informed consent was required.

Consent for publication

Not applicable

\section{Availability of data and materials}

The data supporting the conclusions of this article is included within the article.

\section{Competing interests}

The author declares that there are no competing interests.

\section{Funding}

None declared.

\section{Acknowledgements}

We would like to thank our patients for agreeing to donate their personal data to our study and have these been published.

\section{Authors' contributions}

HGL is fully responsible for this editorial.

\section{Author details}

Women's Hospital, School of Medicine Zhejiang University, 1, Xueshi Road, Hangzhou, Zhejiang 310006, P. R. China.

\section{Abbreviations}

AZF: Azoospermia Factor; ISCN: the International System for Human Cytogenetic Nomenclature guidelines; STS: Six specific sequence-tagged sites; EAA: the European Academy of Andrology; EMGQ: the European Molecular Genetics Quality Network

\section{References}

1. B Peschka, J Leygraaf, K Van der Ven, M Montag, B Schartmann, R Schubert, et al. Type and Frequency of Chromosome Aberrations in 781 Couples Undergoing Intracytoplasmic Sperm Injection. Hum Reprod. 
1999;14(9):2257-2263.

2. Hsu LY. Phenotype/karyotype correlations of $Y$ chromosome aneuploidy with emphasis on structuralaberrations in postnatally diagnosed cases. Am J Med Genet. 1994;53(2):108-140.

3. Siffroi JP, Le Bourhis C, Krausz C, Barbaux S, Quintana-Murci L, Kanafani S, et al. Sex chromosome mosaicism in males carrying $Y$ chromosome long arm deletions. Hum Reprod. 2000; 15(12): 2559-2562.

4. Krausz C, Forti G. Clinical aspects of male infertility. Results Probl Cell Differ. 2000; 28: 1-21.

5. Liu XH, Qiao J, Li R, Yan LY, Chen LX. Y chromosome AZFc microdeletion may not affect the outcomes of ICSI for infertile males with fresh ejaculated sperm. J Assist Reprod Genet. 2013;30(6):813-819.

6. Yuen RK, Merkoulovitch A, MacDonald JR, Vlasschaert M , Lo K, Grober E, et al. Development of a highresolution Y-chromosome microarray for improved male infertility diagnosis. Fertil Steril.2014;101(4): 1079-1085.

7. Li HG, Fan LH, Liu B , Qian YQ, Chen M, Sun YX, et al. The Association Between the Two More Common Genetic Causes of Spermatogenic Failure: A 7-year Retrospective Study. Asian J Androl. 2020,22:1-7.

8. J Mcgowan-Jordan, A Simons, M Schmid. An International System for Human Cytogenetic Nomenclature. Basel: S. Karger press; 2016. p38.

9. Li L, Zhang H, Yang Y, Zhang H, Wang R, Jiang Y, et al. High frequency of $Y$ chromosome microdeletions in male infertility patients with 45,X/46,XY mosaicism. Braz J Med Biol Res. 2020; 53(3):e8980.

10. Rosa RF, D’Ecclesiis WF, Dibbi RP, Rosa RC, Trevisan P, Graziadio C, et al. 45,X/46,XY mosaicism: report on 14 patients from a Brazilian hospital. A retrospective study. Sao Paulo Med J. 2014;132(6): 332-338.

11. Pan Y, Zhang HG, Xi QI, Zhang H, Wang RX, Li LL, et al. Molecular microdeletion analysis of infertile men with karyotypic $Y$ chromosome abnormalities. J Int Med Res. 2018; 46(1):307-315.

12. dos Santos AP, Andrade JG, Piveta CS, de Paulo J, Guerra G Jr, de Mello MP, et al. Screening of $Y$ chromosome microdeletions in $46, X Y$ partial gonadal dysgenesis and in patients with a $45, X / 46, X Y$ karyotype or its variants. BMC Med Genet. 2013;14:115.

13. Peters DG, Yatsenko SA, Surti U, Rajkovic A. Recent advances of genomic testing in perinatal medicine. Semin Perinatol. 2015; 39(1): 44-54.

14. Alvarez-Nava F, Puerta $H$, Soto M, Pineda L, Temponi A. High incidence of $Y$-chromosome microdeletions in gonadal tissues from patients with 45, X/46, XY gonadal dysgenesis. Fertil Steril. 2008, 89(2):458-460.

15. Patsalis PC, Skordis N, Sismani C, Kousoulidou L, Koumbaris G, Eftychi C, et al: Identification of high frequency of $\mathrm{Y}$ chromosome deletions in patients with sex chromosome mosaicism and correlation with the clinical phenotype and Y-chromosome instability. Am J Med Genet A. 2005, 135(2):145-149.

16. Jaruzelska J, Korcz A, Wojda A, Jedrzejczak P, Bierla J, Surmacz T, et al. Mosaicism for 45,X cell line may accentuate the severity of spermatogenic defects in men with AZFc deletion. J Med Genet. 2001; 38(11): 798-802.

17. Patsalis PC, Sismani C, Quintana-Murci L, Taleb-Bekkouche F, Krausz C, McElreavey K. Effects of transmission of $Y$ chromosome AZFc deletions. Lancet. 2002; 360(9341): 1222-1224.

18. Hopps CV, Mielnik A, Goldstein M, Palermo GD, Rosenwaks Z, Schlegel PN. Detection of sperm in men with $Y$ chromosome microdeletions of the AZFa, AZFb and AZFc regions. Hum Reprod. 2003; 18(8): 1660-1665. 
19. Aydemir H, Karkucak M, Cimen HI, Halis F, Kumsar S, Sonbahar AE, et al. A rare combination of $45, \mathrm{X} / 46, \mathrm{XY}$ mosaicism and $\mathrm{Y}$ chromosome microdeletion in an Infertile Man with Azoospermia. Genet Couns. 2016; 27(1): 95-98.

20. Sandra E Kleiman, Leah Yogev, Ofer Lehavi, Ron Hauser, Amnon Botchan, et al. The likelihood of finding mature sperm cells in men with AZFb or AZFb-c deletions: six new cases and a review of the literature (1994-2010). Fertil Steril. 2011;95(6):2005-2012. 